\title{
A Shear Lag Analysis for Composite Box Girders with Deformable Connectors
}

V. Křístek

A method is proposed for shear lag analysis which can be applied to steel-concrete composite box girders. The proposed method uses harmonic analysis and allows the determination of shear lag effects from simple calculations so that the method is regarded as a design aid. The character of the method can illustrate the influence of certain key parameters upon the extent of the shear lag effect.

Keywords: shear lag, composite girder, stress distribution, harmonic analysis.

\section{Introduction}

The effects of shear lag can cause a significant increase in the longitudinal stresses developed in steel box girders. Previous investigations have shown that the extent of shear lag within a flange plate is dependent on the ratio between the axial stiffness and the shear stiffness of the plate. The introduction of longitudinal stiffeners increases the axial stiffness without changing the shear stiffness so that there is a consequent increase in shear lag. Stiffeners are, of course, introduced to increase the resistance of the compressed flange to buckling. It has been proven in [1] that it is far more advantageous, from the point of view of shear lag, if the flange plate is stiffened with a layer of concrete that is made to act compositely with the steel plate (Fig.1a). The necessary composite action can be achieved by means of shear studs welded to the steel plate.

Among many applications of composite arrangements, the case of increasing the load carrying capacity of an existing steel box girder bridge may be mentioned as a special example. The bottom flange plate in the hogging moment regions over the internal supports of a continuous girder is particularly susceptible to the effects of shear lag. The most

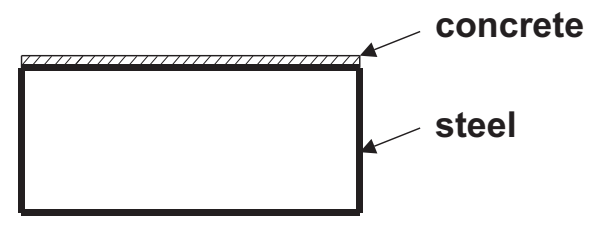

(a)

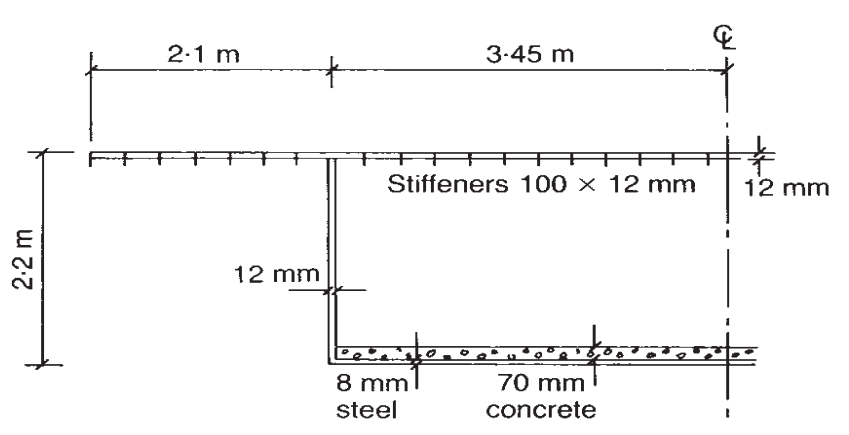

(b)

Fig. 1: (a) Steel-concrete composite girder - simplified form of cross section, (b) concrete layer added to the compression bottom flange plate in the hogging moment regions obvious way of strengthening these regions is to weld on more longitudinal stiffeners in the compression zone of the bottom flange. This will increase the buckling resistance of the flange, but it will also accentuate the shear lag problem. An alternative method of strengthening an existing bridge girder is to add a concrete layer to the compression flange so that it acts compositely with the steel (Fig. 1b), which will increase the buckling resistance while also controlling the shear lag effect. Although the method is applicable primarily for strengthening an existing bridge, it may well provide an economic alternative in the design of a new box girder.

A perfect connection between the steel flange and the concrete layer exists, however, only theoretically. Although there certainly will be an intention to benefit from full composite interaction, the studs placed at regular distances, which are commonly used as connectors at the present time, exhibit some unavoidable deformability.

\section{Governing equations}

Shear flows ${ }_{s} q$ and ${ }_{c} q$, and normal forces ${ }_{s} n_{x}$ and ${ }_{c} n_{x}$ per unit width act on a typical element of the steel flange sheet or the concrete layer, respectively (see Fig. 2).

The equations governing the equilibrium in the longitudinal direction are:

for the steel sheet (Fig. 2a)

$$
\frac{\partial_{s} n_{x}}{\partial x}+\frac{\partial_{s} q}{\partial y}+f=0
$$

for the concrete layer (Fig. 2b)

$$
\frac{\partial_{c} n_{x}}{\partial x}+\frac{\partial_{c} q}{\partial y}-f=0,
$$

in which $f$ is the shear acting in the longitudinal direction at the interface between the steel flange and the concrete layer.

If the contribution of small traverse forces to the strains is neglected, it may be written:

$$
\begin{aligned}
& { }_{s} \varepsilon_{x}=\frac{\partial_{s} u}{\partial x}=\frac{{ }_{s} n_{x}}{t_{s} E_{s}}, \\
& { }_{c} \varepsilon_{x}=\frac{\partial_{c} u}{\partial x}=\frac{{ }_{c} n_{x}}{t_{c} E_{c}}, \\
& { }_{s} \varepsilon_{y}=-v_{s} \varepsilon_{s} \varepsilon_{x}=-v_{s} \frac{{ }_{s} n_{x}}{t_{s} E_{s}}, \\
& { }_{c} \varepsilon_{y}=-v_{c}{ }_{c} \varepsilon_{x}=-v_{c} \frac{{ }_{c} n_{x}}{t_{c} E_{s}},
\end{aligned}
$$


a) the concrete sheet

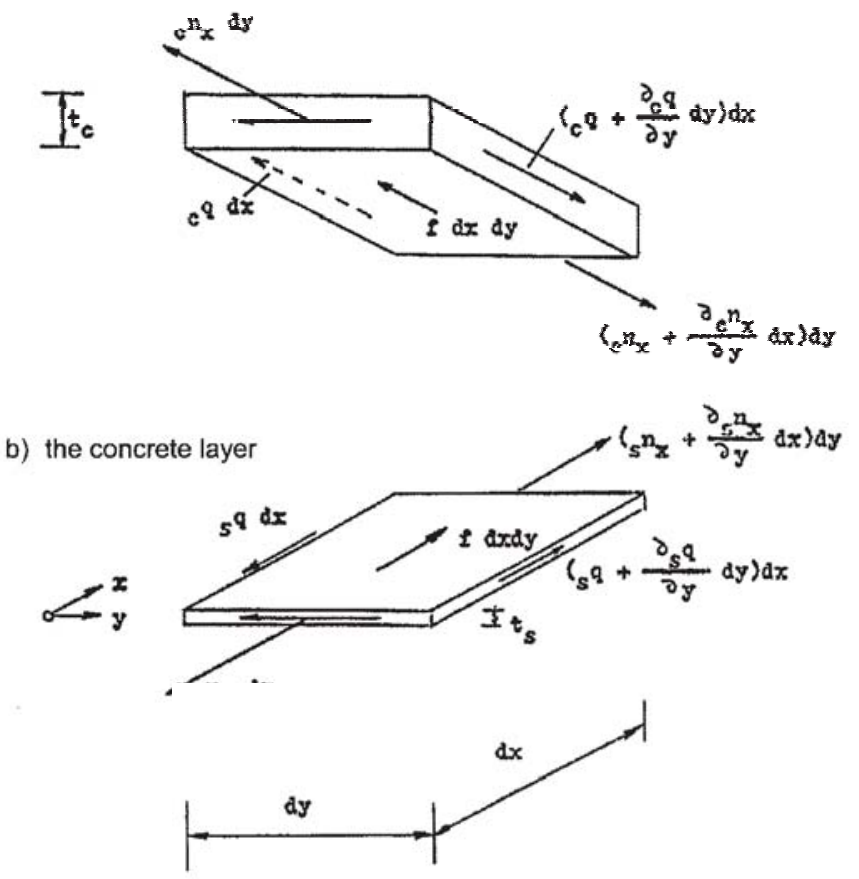

Fig. 2: Equilibrium conditions in the longitudinal direction

$$
\begin{aligned}
& \gamma_{s}=\frac{{ }_{s} q}{t_{s} G_{s}}, \\
& \gamma_{c}=\frac{{ }_{c} q}{t_{c} G_{c}},
\end{aligned}
$$

where $t_{s}$ and $t_{c}$ are the thicknesses of the steel flange and the concrete layer, respectively, $\varepsilon_{x}$ and $\varepsilon_{y}$ are the direct strains in the longitudinal and transverse directions, respectively, and $\gamma$ are the shear strains. E, $G$ and $v$ represent Young's moduli, the shear moduli and Poisson's ratios, respectively; $u$ are the longitudinal displacements. follows:

The general form of the condition of compatibility is as

$$
\frac{\partial^{2} \varepsilon_{x}}{\partial y^{2}}+\frac{\partial^{2} \varepsilon_{y}}{\partial x^{2}}=\frac{\partial^{2} \gamma}{\partial x \partial y} .
$$

Substituting the strains from Eqs. (3)-(8) it is obtained: for the steel:

$$
\frac{\partial^{2}{ }_{s} n_{x}}{\partial y^{2}}-v_{s} \frac{\partial^{2}{ }_{s} n_{x}}{\partial x^{2}}=2\left(1+v_{s}\right) \frac{\partial^{2}{ }_{s} q}{\partial x \partial y},
$$

for the concrete layer:

$$
\frac{\partial^{2}{ }_{c} n_{x}}{\partial y^{2}}-v_{c} \frac{\partial^{2}{ }_{c} n_{x}}{\partial x^{2}}=2\left(1+v_{c}\right) \frac{\partial^{2}{ }_{c} q}{\partial x \partial y} .
$$

Substituting for the shear flows ${ }_{s} q$ and ${ }_{c} q$ from equations (1) and (2):

$$
\begin{aligned}
& \frac{\partial^{2}{ }_{s} n_{x}}{\partial y^{2}}+\left(2+v_{s}\right) \frac{\partial^{2}{ }_{s} n_{x}}{\partial x^{2}}+2\left(1+v_{s}\right) \frac{\partial f}{\partial x}=0, \\
& \frac{\partial^{2}{ }_{c} n_{x}}{\partial y^{2}}+\left(2+v_{c}\right) \frac{\partial^{2}{ }_{c} n_{x}}{\partial x^{2}}-2\left(1+v_{c}\right) \frac{\partial f}{\partial x}=0 .
\end{aligned}
$$

It may be assumed that the shear $f$ acting between the steel sheet and the concrete layer, being provided by deformable connectors, is proportional to the mutual longitudinal slip which occurs at the interface between the two components, i.e.

$$
f=k\left({ }_{s} u-{ }_{c} u\right)
$$

where $k$ is the connector stiffness.

Eq. (14) may be written in the form:

$$
\frac{\partial f}{\partial x}=k\left(\frac{\partial_{s} u}{\partial x}-\frac{\partial_{c} u}{\partial x}\right)=k\left(\frac{{ }_{s} n_{x}}{t_{s} E_{s}}-\frac{{ }_{c} n_{x}}{t_{c} E_{c}}\right) .
$$

The following Fourier series may express the searched functions:

$$
\begin{aligned}
{ }_{s} n_{x} & =\sum_{s} N_{j}(y) \sin \frac{j \pi x}{L}, \\
{ }_{c} n_{x} & =\sum_{c} N_{j}(y) \sin \frac{j \pi x}{L}, \\
f & =\sum_{j=1}^{\infty} F_{j}(y) \cos \frac{j \pi x}{L},
\end{aligned}
$$

where $L$ is the effective span-length.

Eqs. (12), (13) and (15) can be written in the form:

$$
\begin{gathered}
{ }_{s} \ddot{N}_{j}-\frac{j^{2} \pi^{2}}{L^{2}}\left(2+v_{s}\right){ }_{s} N_{j}-\frac{2 j \pi}{L}\left(1+v_{s}\right) F_{j}=0, \\
{ }_{c} \ddot{N}_{j}-\frac{j^{2} \pi^{2}}{L^{2}}\left(2+v_{c}\right){ }_{c} N_{j}-\frac{2 j \pi}{L}\left(1+v_{c}\right) F_{j}=0, \\
\frac{j \pi}{L} F_{j}+k\left(\frac{{ }_{s} N_{j}}{t_{s} E_{s}}-\frac{{ }_{c} N_{j}}{t_{c} E_{c}}\right)=0,
\end{gathered}
$$

in which ${ }_{s} \ddot{N}_{j}=\frac{\partial^{2}{ }_{s} N_{j}}{\partial y^{2}}$, etc.

These relations represent a set of three equations for the unknown functions ${ }_{s} N_{j}(y),{ }_{c} N_{j}(y)$ and $F_{j}(y)$, which can be adjusted to the following system of two differential equations

$$
\begin{aligned}
& { }_{s} \ddot{N}_{j}+a_{j s} N_{j}-c_{j c} N_{j}=0, \\
& { }_{c} \ddot{N}_{j}+b_{j c} N_{j}-d_{j s} N_{j}=0,
\end{aligned}
$$

where

$$
\begin{aligned}
& a_{j}=-\frac{j^{2} \pi^{2}}{L^{2}}\left(2+v_{s}\right)+\frac{2 k\left(1+v_{s}\right)}{t_{s} E_{s}}, \\
& b_{j}=-\frac{j^{2} \pi^{2}}{L^{2}}\left(2+v_{c}\right)+\frac{2 k\left(1+v_{c}\right)}{t_{c} E_{c}}, \\
& c_{j}=\frac{2 k\left(1+v_{s}\right)}{t_{c} E_{c}}, \\
& d_{j}=\frac{2 k\left(1+v_{c}\right)}{t_{s} E_{s}} .
\end{aligned}
$$

It follows from Eq. (22) that

$$
{ }_{c} N_{j}=\frac{1}{c_{j}}\left({ }_{s} \ddot{N}_{j}+a_{j s} N_{j}\right),
$$


which substituted into Eq. (23) allows to obtain a differential equation of the fourth order

$\frac{\mathrm{d}^{4}{ }_{s} N_{j}(y)}{\mathrm{d} y^{4}}+A_{j} \frac{\mathrm{d}^{2}{ }_{s} N_{j}(y)}{\mathrm{d} y^{2}}+B_{j}{ }_{s} N_{j}(y)=0$,

whose coefficients are

$$
\begin{aligned}
A_{j} & =-\frac{j^{2} \pi^{2}}{L^{2}}\left(4+v_{s}+v_{c}\right)+2 k\left[\frac{\left(1+v_{s}\right)}{t_{s} E_{s}}+\frac{\left(1+v_{c}\right)}{t_{c} E_{c}}\right], \\
B_{j}^{2} & =\frac{j^{2} \pi^{2}}{L^{2}}\left\{\frac{j^{2} \pi^{2}}{L^{2}}\left(2+v_{s}\right)\left(2+v_{c}\right)+\right. \\
& \left.+2 k\left[\frac{\left(1+v_{s}\right)\left(2+v_{c}\right)}{t_{s} E_{s}}+\frac{\left(1+v_{c}\right)\left(2+v_{s}\right)}{t_{c} E_{c}}\right]\right\} .
\end{aligned}
$$

The general solution of (26), if the case of complex roots of the characteristic equation is assumed, is

$$
\begin{aligned}
{ }_{s} N_{j}(y) & =C_{1, j} P_{1, j}(y)+C_{2, j} P_{2, j}(y)+ \\
& +C_{3, j} P_{3, j}(y)+C_{4, j} P_{4, j}(y),
\end{aligned}
$$

where

$$
\begin{aligned}
& P_{1, j}(y)=\sinh \xi_{j} y \sin \eta_{j} y \\
& P_{2, j}(y)=\cosh \xi_{j} y \cos \eta_{j} y \\
& P_{3, j}(y)=\cosh \xi_{j} y \sin \eta_{j} y \\
& P_{4, j}(y)=\sinh \xi_{j} y \cos \eta_{j} y
\end{aligned}
$$

and

$$
\xi_{j}=\sqrt{\frac{B_{j}}{2}-\frac{A_{j}}{4}}, \quad \eta_{j}=\sqrt{\frac{B_{j}}{2}+\frac{A_{j}}{4}} .
$$

The amplitude function ${ }_{c} N_{j}(y)$, according to Eq. (25), is determined as

$$
\begin{aligned}
{ }_{c} N_{j}(y)=\frac{1}{c_{j}} & \left\{C_{1, j}\left[r_{j} P_{1, j}(y)+s_{j} P_{2, j}(y)\right]+\right. \\
& +C_{2, j}\left[r_{j} P_{2, j}(y)-s_{j} P_{1, j}(y)\right]+ \\
& +C_{3, j}\left[r_{j} P_{3, j}(y)+s_{j} P_{4, j}(y)\right]+ \\
& \left.+C_{4, j}\left[r_{j} P_{4, j}(y)-s_{j} P_{3, j}(y)\right]\right\},
\end{aligned}
$$

in which

$$
\begin{aligned}
& r_{j}=\xi_{j}^{2}-\eta_{j}^{2}+a_{i}, \\
& s_{j}=2 \xi_{j} \eta_{j} .
\end{aligned}
$$

\section{Boundary and loading conditions}

Shear lag analysis is carried out for loads, placed symmetrically on the girder cross-section. Thus, assuming the origin of the traverse co-ordinate $y$ to be taken at the mid-width of the flange, i. e. at the axis of symmetry, then, because of the symmetry

$$
C_{3, j}=C_{4, j}=0
$$

so that from equations (28) and (30), the distributions across the flange width of the normal forces in the steel flange and in the concrete layer are governed by:

$$
\begin{gathered}
{ }_{s} N_{j}(y)=C_{1, j} P_{1, j}(y)+C_{2, j} P_{2, j}(y) \\
{ }_{c} N_{j}(y)=\frac{1}{c_{j}}\left\{C_{1, j}\left[r_{j} P_{1, j}(y)+s_{j} P_{2, j}(y)\right]+\right. \\
\left.+C_{2, j}\left[r_{j} P_{2, j}(y)-s_{j} P_{1, j}(y)\right]\right\} .
\end{gathered}
$$

The amplitude function governing the distribution of the shear at the interface between the steel and concrete can be expressed from equation (21) as

$$
\begin{aligned}
F_{j}(y)=\frac{k L}{j \pi} & \left\{C _ { 1 , j } \left\langle\frac{1}{c_{j} t_{c} E_{c}}\left[r_{j} P_{1, j}(y)+s_{j} P_{2, j}(y)\right]-\right.\right. \\
& \left.-\frac{P_{1, j}(y)}{t_{s} E_{s}}\right\rangle+ \\
& +C_{2, j}\left\langle\frac{1}{c_{j} t_{c} E_{c}}\left[r_{j} P_{2, j}(y)-s_{j} P_{1, j}(y)\right]-\right. \\
& \left.\left.-\frac{P_{2, j}(y)}{t_{s} E_{s}}\right\rangle\right\} .
\end{aligned}
$$

It is seen that also this distribution is symmetrical about the flange mid-width.

The values of the remaining constants $C_{1, j}$ and $C_{2, j}$ can be determined from the shear loading conditions at the edges of the steel flange and the concrete layer.

Combining equations (1), (16), (18):

$$
\begin{aligned}
\frac{\partial_{s} q}{\partial y}= & -\frac{\partial_{s} n_{x}}{\partial x}-f=\sum_{j=1}^{\infty}\left[\frac{j \pi}{L}{ }_{s} N_{j}(y)+F_{j}(y)\right] \cos \frac{j \pi x}{L}= \\
= & \sum_{j=1}^{\infty}\left\{C _ { 1 , j } \left\langle\left[\frac{j \pi}{L}+\frac{k L}{j \pi}\left(\frac{r_{j}}{c_{j} t_{c} E_{c}}-\frac{1}{t_{s} E_{s}}\right)\right] P_{1, j}(y)+\right.\right. \\
& \left.+\frac{k L s_{j}}{j \pi c_{j} t_{c} E_{c}} P_{2, j}(y)\right\rangle+ \\
& +C_{2, j}\left\langle\left[\frac{j \pi}{L}+\frac{k L}{j \pi}\left(\frac{r_{j}}{c_{j} t_{c} E_{c}}-\frac{1}{t_{s} E_{s}}\right)\right] P_{2, j}(y)-\right. \\
& \left.\left.-\frac{k L s_{j}}{j \pi c_{j} t_{c} E_{c}} P_{1, j}(y)\right\rangle\right\} \cos \frac{j \pi x}{L}
\end{aligned}
$$

so that (by integrating with respect to $y$ ) the shear flow in the steel flange at any point may be expressed as:

$$
\begin{aligned}
{ }_{s} q(x, y)= & \sum_{j=1}^{\infty} \frac{1}{\xi_{j}^{2}+\eta_{j}^{2}}\left\{C _ { 1 , j } \left\langle\left[\frac{j \pi}{L}+\frac{k L}{j \pi}\left(\frac{r_{j}}{c_{j} t_{c} E_{c}}-\frac{1}{t_{s} E_{s}}\right)\right] \times\right.\right. \\
& \times\left[\xi_{j} P_{3, j}(y)-\eta_{j} P_{4, j}(y)\right]+ \\
& \left.+\frac{k L s_{j}}{j \pi c_{j} t_{c} E_{c}}\left[\xi_{j} P_{4, j}(y)+\eta_{j} P_{3, j}(y)\right]\right\rangle+ \\
& +C_{2, j}\left\langle\left[\frac{j \pi}{L}+\frac{k L}{j \pi}\left(\frac{r_{j}}{c_{j} t_{c} E_{c}}-\frac{1}{t_{s} E_{s}}\right)\right] \times\right. \\
& \times\left[\xi_{j} P_{4, j}(y)+\eta_{j} P_{3, j}(y)\right]- \\
& \left.-\frac{k L s_{j}}{j \pi c_{j} t_{c} E_{c}}\left[\xi_{j} P_{3, j}(y)-\eta_{j} P_{4, j}(y)\right]\right\rangle \cos \frac{j \pi x}{L}= \\
& =-\sum_{j=1}^{\infty}\left[C_{1, j} Z_{1, j}(y)+C_{2, j} Z_{2, j}(y)\right] \cos \frac{j \pi x}{L} .
\end{aligned}
$$


Similarly, the shear flow in the concrete layer, combining equations (2), (17) and (18), is governed by the following relation:

$$
\begin{aligned}
\frac{\partial_{c} q}{\partial y} & =-\frac{\partial_{c} n_{x}}{\partial x}+f=-\sum_{j=1}^{\infty}\left[\frac{j \pi}{L}{ }_{c} N_{j}(y)-F_{j}(y)\right] \cos \frac{j \pi x}{L}= \\
& =-\sum_{j=1}^{\infty}\left\{C _ { 1 , j } \left\langle\left(\frac{j \pi}{L c_{j}}+\frac{k L}{j \pi c_{j} t_{c} E_{c}}\right)\left[r_{j} P_{1, j}(y)+s_{j} P_{2, j}(y)\right]+\right.\right. \\
& \left.+\frac{k L}{j \pi t_{s} E_{s}} P_{1, j}(y)\right\rangle+ \\
& +C_{2, j}\left(\left(\frac{j \pi}{L c_{j}}+\frac{k L}{j \pi c_{j} t_{c} E_{c}}\right)\left[r_{j} P_{2, j}(y)-s_{j} P_{1, j}(y)\right]+\right. \\
& \left.\left.+\frac{k L s_{j}}{j \pi t_{s} E_{s}} P_{2, j}(y)\right)\right\} \cos \frac{j \pi x}{L} .
\end{aligned}
$$

Thus, the shear flow in the concrete layer at any point is expressed as:

$$
\begin{aligned}
{ }_{c} q(x, y) & =-\sum_{j=1}^{\infty} \frac{1}{\xi_{j}^{2}+\eta_{j}^{2}}\left\{C _ { 1 , j } \left\langle\left(\frac{j \pi}{L c_{j}}-\frac{k L}{j \pi c_{j} t_{c} E_{c}}\right) \times\right.\right. \\
& \times\left(r_{j}\left[\xi_{j} P_{3, j}(y)-\eta_{j} P_{4, j}(y)\right]+\right. \\
& \left.+s_{j}\left[\xi_{j} P_{4, j}(y)+\eta_{j} P_{3, j}(y)\right]\right)+ \\
& \left.+\frac{k L}{j \pi t_{s} E_{s}}\left[\xi_{j} P_{3, j}(y)-\eta_{j} P_{4, j}(y)\right]\right\rangle+ \\
& +C_{2, j} /\left(\frac{j \pi}{L c_{j}}-\frac{k L}{j \pi c_{j} t_{c} E_{c}}\right) \times \\
& \times\left(r_{j}\left[\xi_{j} P_{4, j}(y)+\eta_{j} P_{3, j}(y)\right]-\right. \\
& \left.-s\left[\xi_{j} P_{3, j}(y)-\eta_{j} P_{4, j}(y)\right]\right)+ \\
& \left.+\frac{k L}{j \pi t_{s} E_{s}}\left[\xi_{j} P_{4, j}(y)+\eta_{j} P_{3, j}(y)\right]\right\rangle \cos \frac{j \pi x}{L}
\end{aligned}
$$

which may be written in the form

$$
\begin{aligned}
{ }_{c} q(x, y) & =-\sum_{j=1}^{\infty} \frac{1}{\xi_{j}^{2}+\eta_{j}^{2}}\left\{C _ { 1 , j } \left\langle\frac{1}{c_{j}}\left(\frac{j \pi}{L}-\frac{k L}{j \pi t_{c} E_{c}}\right) \times\right.\right. \\
& \times\left[\left(r_{j} \xi_{j}+s_{j} \eta_{j}\right) P_{3, j}(y)+\left(s_{j} \xi_{j}-r_{j} \eta_{j}\right) P_{4, j}(y)\right]+ \\
& \left.+\frac{k L}{j \pi t_{s} E_{s}}\left[\xi_{j} P_{3, j}(y)-\eta_{j} P_{4, j}(y)\right]\right\rangle+ \\
& +C_{2, j}\left(\frac{1}{c_{j}}\left(\frac{j \pi}{L}-\frac{k L}{j \pi t_{c} E_{c}}\right) \times\right. \\
& \times\left[\left(r_{j} \eta_{j}-s_{j} \xi_{j}\right) P_{3, j}(y)+\left(r_{j} \xi_{j}+s_{j} \eta_{j}\right) P_{4, j}(y)\right]+ \\
& \left.+\frac{k L}{j \pi t_{s} E_{s}}\left[\eta_{j} P_{3, j}(y)+\xi_{j} P_{4, j}(y)\right]\right\rangle \cos \frac{j \pi x}{L}= \\
& =-\sum_{j=1}^{\infty}\left[C_{1, j} Z_{3, j}(y)+C_{2, j} Z_{4, j}(y)\right] \cos \frac{j \pi x}{L} .
\end{aligned}
$$

From simple beam theory, the shear flow $q_{e}(x)$ transmitted from the web to the edge of the steel flange can be approximated as

$$
q_{e}(x)=V(x) \frac{\left(t_{s}+t_{c} \frac{E_{c}}{E_{s}}\right) b e}{2 I},
$$

where $V(x)$ is the total shear force acting on the beam cross-section at position $x$; $I$ is the second moment of area of the composite cross-section (the contribution of the concrete layer being reduced by the ratio $\left.E_{c} / E_{s}\right), e$ is the distance from the cross-sectional neutral axis to the centroid of the composite flange.

The shear flow transmitted at the edge of the flange can also be expressed in the form of the Fourier series. For the case of simply supported ends, the series takes the form

$$
q_{e}(x)=\sum_{j=1}^{\infty} Q_{e, j} \cos \frac{j \pi x}{L},
$$

where

$$
\begin{aligned}
Q_{e, j} & =\frac{2}{L} \int_{0}^{L} q_{e}(x) \cos \frac{j \pi x}{L} \mathrm{~d} x= \\
& =\frac{\left(t_{s}+t_{c} \frac{E_{c}}{E_{b}}\right) b e}{I L} \int_{0}^{L} V(x) \omega \frac{j \pi x}{L} \mathrm{~d} x .
\end{aligned}
$$

Values of the coefficients $Q_{e, j}$, evaluated according to this formula, are listed in Table 1 for a few typical cases. that

It must hold at the joint of the web and the steel flange

$$
{ }_{s} q\left(x, \frac{b}{2}\right)=q_{e}(x)
$$

and at the edge of the concrete layer that

$$
{ }_{s} q\left(x, \frac{b}{2}\right)=0
$$

since the concrete layer is not directly connected to the web.

Combining relations (37), (39), (41), (43) and (44), it is possible to form two equations to determine the constants $C_{1, j}$ and $C_{2, j}$, which can be written in matrix form as:

$$
\left[\begin{array}{cc}
Z_{1, j}\left(\frac{b}{2}\right) & Z_{2, j}\left(\frac{b}{2}\right) \\
Z_{3, j}\left(\frac{b}{2}\right) & Z_{4, j}\left(\frac{b}{2}\right)
\end{array}\right]\left\{\begin{array}{c}
C_{1, j} \\
C_{2, j}
\end{array}\right\}=\left\{\begin{array}{c}
Q_{e, j} \\
0
\end{array}\right\}
$$

\section{Completion of shear lag analysis}

Having thus determined the two constants, the amplitudes of the normal longitudinal forces ${ }_{s} N_{j}$ a ${ }_{c} N_{j}$ for any particular harmonic can be obtained from equations (33) and (34). The magnitudes of these forces varies across the width of the flange; the peak value of the normal longitudinal force ${ }_{s} N_{j}(y)$ acting in the steel sheet occurs at the edge, i. e. where $y=b / 2$. The distribution of the normal longitudinal force ${ }_{c} N_{j}(y)$ across the width of the concrete layer may have a more general character. 
Table 1: Values of the coefficient $Q_{e, j}$ for different tapes of loading

\begin{tabular}{|c|c|c|}
\hline \multirow{2}{*}{ Type of loading } & \multicolumn{2}{|c|}{$Q_{e}, j$} \\
\hline & Section $3.1 .4\left(E_{2} \cdot(3.24)\right)$ & Section 3.1 .5 \\
\hline $\begin{array}{l}\text { Uniformly distributed } \\
\text { loading } \mathrm{W}\end{array}$ & $2 \frac{L w \bar{t} b e}{j^{2} \pi^{2} I}$ & $2 \frac{L w\left(t b+\Sigma A_{s}\right) e}{j^{2} \pi^{2} I}$ \\
\hline $\begin{array}{l}\text { Force } P \text { distributed over } \\
\text { length } \delta \text { (symmetrical } \\
\text { about mid-spon) }\end{array}$ & $2 \frac{P L \bar{t} b e}{\delta j^{2} \pi^{2} I} \sin \frac{j \pi}{2} \sin \frac{j \pi \delta}{2 L}$ & $2 \frac{P L\left(t b+\Sigma A_{s}\right) e}{\delta j^{2} \pi^{2} I} \sin \frac{j \pi}{2} \sin \frac{j \pi \delta}{2 L}$ \\
\hline $\begin{array}{l}\text { Force } P \text { distributed over } \\
\text { length } \delta \\
\text { (at general position) }\end{array}$ & $\begin{array}{r}2 \frac{\rho L \bar{t} b e}{\delta j^{2} \pi^{2} I} \sin \frac{j \pi \eta}{L} \sin \frac{j \pi \delta}{2 L} \\
\quad\left(\text { for } \frac{\delta}{2} \leq\right.\end{array}$ & $\begin{array}{l}2 \frac{P L\left(t b+\Sigma A_{s}\right) e}{\delta j^{2} \pi^{2} I} \sin \frac{j \pi \eta}{L} \sin \frac{j \pi \delta}{2 L} \\
\left.\leq L-\frac{\delta}{2}\right)\end{array}$ \\
\hline
\end{tabular}

Knowing the amplitudes ${ }_{s} N_{j}$ and ${ }_{c} N_{j}$, the values of the longitudinal normal forces per unit width ${ }_{s} n_{x}(x, y)$ and ${ }_{c} n_{x}(x, y)$ may be determined from equations (16) and (17) for any position on the flange. Also the shear flows ${ }_{s} q(x, y)$ and ${ }_{c} q(x, y)$ at any point may be determined from equations $(37)$ and (39).

To evaluate the forces taken by the studs, the shear $f$ acting at the interface between the steel flange and the concrete layer is to be determined according to equation (18). The amplitude function $F_{j}(y)$, describing the distribution of the shear across the flange width, is determined - knowing constants $C_{1, j}$ and $C_{2, j}$ - by equation (35).

\section{Summary of calculations}

For any particular girder with composite flanges, the first step in the calculation of the shear lag effect is to determine the value of coefficient $Q_{e, j}$ from equation (42). The value $Q_{e, j}$ is then substituted into the right-hand side of equations (45) to give the values of constants $C_{1, j}$ and $C_{2, j}$, and, finally, for any harmonic the amplitudes of all the functions are required. These, in turn, are substituted into equations (16), (17), (18), (37) and (39) to give the normal forces per unit width, the shear acting at the interface between the steel and concrete components, and the shear flows at any position on the composite flange.

The corresponding value of the longitudinal stress in the steel component of the flange is then given by:

$$
\sigma_{s x}(x, y)=\frac{{ }_{s} n_{x}(x, y)}{t_{s}}
$$

and the longitudinal stress in the concrete layer is obtained as:

$$
\sigma_{c x}(x, y)=\frac{{ }_{c} n_{x}(x, y)}{t_{c}}
$$

Should the shear stress values also be required, then, having evaluated the shear flows ${ }_{s} q(x, y)$ and ${ }_{c} q(x, y)$ at any point, the shearing stress in the steel is obtained as:

$$
\tau_{s x}(x, y)=\frac{{ }_{s} q(x, y)}{t_{s}}
$$

and the shear stress in the concrete layer is given by:

$$
\tau_{c x}(x, y)=\frac{{ }_{c} q(x, y)}{t_{c}}
$$

\section{Conclusions}

This paper has described the development of an approximate analytical method for analysing the stress distribution in the flanges of composite steel-concrete beams with deformable connectors. Its primary advantage is the closed form of the results obtained and its ease of application. The method is also very suitable for parametric studies investigating the influences of various arrangements, and for optimisation studies.

To conclude, it should be noted that - besides the mechanical effects - the thermal effects can also play an important role in the structural performance of steel-concrete composite beams, see, e.g., [2].

\section{Acknowledgment}

Support for this research through grants 103/02/.1005 and 103/02/.0020 from the Grant Agency of the Czech Republic is gratefully acknowledged. 


\section{References}

[1] Kř́stek V., Evans H. R.: "A hand calculation of the shear lag effect in unstiffened flanges and in flanges with closely spaced stiffeners." Civil Engng Practising Design Engrs, Vol. 4 (1985) No. 2.

[2] Ř́mal J.: "Measurement of Temperature Fields in Composite Steel and Concrete Bridges." CTU Publishing House, Prague, 2003.

[3] Kř́stek V. Evans H. R., Ahmad M. K. M.: "A Shear Lag Analysis for Composite Box Girders." J. Construct. Steel Research, Vol. 16 (1990).

[4] Evans H. R., Ahmad M. K. M., Křístek V.: "Shear lag in composite box girders of complex cross sections". J. Construct. Steel Research, Vol. 24 (1993).

[5] Kř́stek V., Studnička J.: "Composite girders with deformable connection between steel and concrete."
(Chap. in Composite Steel-Concrete Structures), Applied Science Publishers, Elsevier, London 1988, Edited by R. Narayanan.

[6] Evans H. R., Křístek V., Škaloud M.: "Strengthening steel box girder bridges by controlling the effects of shear lag." Proc. Instn. Civ. Engrs. Structs. and Bldgs., Vol. 104 (Nov. 1994).

Prof. Ing. Vladimír Křístek, DrSc.

phone: +420224353875

e-mail: kristek@fsv.cvut.cz

Department of Concrete Structures and Bridges

Czech Technical University in Prague

Faculty of Civil Engineering

Thákurova 7

Praha 6, Czech Republic 